\title{
OpenEHR modeling: improving clinical records during the COVID-19 pandemic
}

\author{
Daniela Oliveira ${ }^{2} \cdot$ Rui Miranda $^{2} \cdot$ Pedro Leuschner $^{2} \cdot \mathrm{Nuno} \mathrm{Abreu}^{2} \cdot$ Manuel Filipe Santos $^{2} \cdot$ Antonio Abelha $^{2} \cdot$ \\ José Machado ${ }^{1}$ (1)
}

Received: 12 January 2021 / Accepted: 26 April 2021 / Published online: 4 May 2021

(c) IUPESM and Springer-Verlag GmbH Germany, part of Springer Nature 2021

\begin{abstract}
The COVID-19 pandemic had put pressure on various national healthcare systems, due to the lack of health professionals and exhaustion of those avaliable, as well as lack of interoperability and inability to restructure their IT systems. Therefore, the restructuring of institutions at all levels is essential, especially at the level of their information systems. Furthermore, the COVID-19 pandemic had arrived in Portugal at March 2020, with a breakout on the northern region. In order to quickly respond to the pandemic, the CHUP healthcare institution, known as a research center, has embraced the challenge of developing and integrating a new approach based on the openEHR standard to interoperate with the institution's existing information and its systems. An openEHR clinical modelling methodology was outlined and adopted, followed by a survey of daily clinical and technical requirements. With the arrival of the virus in Portugal, the CHUP institution has undergone through constant changes in their working methodologies as well as their openEHR modelling. As a result, an openEHR patient care workflow for COVID-19 was developed.
\end{abstract}

Keywords COVID-19 $\cdot$ OpenEHR $\cdot$ Archetypes $\cdot$ Templates $\cdot$ Interoperability $\cdot$ Healthcare information systems $\cdot$ Electronic health record

\section{Introduction}

Interoperability is increasingly viewed as an essential aspect of an HIS. A complete and understandable EHR - one that includes all the relevant clinical information concerning a single patient throughout her/his life - aggregates different sources of medical data, such as progress notes, lab data and exam reports, inpatient care or surgery reports. In most modern hospitals, data is recorded in multiple discrete systems with limited interoperability. To present this information in a logic, readable way, considerable effort must be placed in 'bridging' these systems. Clinical data has a

This article is part of the COVID-19 Health Technology: Design, Regulation, Management, Assessment

José Machado

jmac@di.uminho.pt

1 Escola de Engenharia, Universidade do Minho, Campus Azurum, Guimarues 4800-058, Portugal

2 Centro Hospitalar Universitario do Porto, Largo do Prof. Abel Salazar 4800-058, Porto, Portugal complex structure and meaning. Moreover, most HIS were incrementally built by adding up new modules, each one of them aiming to solve a particular need of single units or departments within the hospital, often disregarding interoperability. As a result, to develop basic functions like information exchanging and sharing is becoming more and more demanding, consuming precious resources. As a result the HIS can become increasingly fractured, paving the way to cumbersome outcomes: increasing probability of clinical errors, rising costs for the institution, and decreasing the overall quality of healthcare [1-4].

Thus, the main goal of an HIS is to support the continuity of care across multiple institutions and healthcare providers in a ubiquitous, interoperable, and secure way, so as to facilitate data collection, aggregation, and analysis. This is the basis to provide a completer and more coherent picture of the patient, allowing for better clinical judgment and safer decisions. Furthermore, CDS systems often rely on the same foundations, to achieve its role as a useful, reliable decision aid at the point of care. The first COVID-19 outbreak began in mainland China, and quickly proliferated to the rest of the world, causing a global pandemic situation [5]. 
The current epidemic situation has overloaded healthcare systems in many countries. This was due to numerous factors such as insufficient human and material resources, in face of an increased health care demand. On top of that, difficulties experienced sharing data on normal day-to-day work were enhanced by the pressure caused by this pandemic, causing more burden to health professionals. In these emergency times, clinical data produced is a precious asset useful not only for clinical decisions but for health political governance, providing updated and on real time information regarding the pandemic's evolution. In order to optimize the workflow for the treatment of patients with COVID-19 in Portugal, the CHUP institution has developed a new system based on openEHR specification. Thus, a hybrid solution that interoperated with existing systems was developed, ensuring quick and effective communication protocols between users with minimum effort. The next subchapters will offer key basic definitions for the development of the system under study.

\subsection{Interoperability in healthcare}

In literature, one can find multiple definitions for interoperability. In short, clinical data interoperability is the capacity of two or more systems to interpret data exchanged or shared between them. Each interoperability level has specific syntactic, semantic, and cross-domain requirements. International e-health communities have been advising for and working on the use of clinical information models as a mean to increase interoperability $[1,6,7]$. The CEN advocates that proper use of standards is warranted if one aims to build more reliable and efficient software, while increasing connectivity with other appliances and components. Thus, healthcare interoperability can be ensured using IT standards, with the aim to harmonize and enable interoperability with external applications as much as possible [8].

Semantic interoperability is critical for the continuous improvement of patient's quality of care, global health research, and the management of healthcare institutions. It ensures the preservation of meaning while exchanging information is exchanged between different systems [9]. The interdependence of technical and domain-knowledge is recognized as a barrier to successfully implementing and regularly updating an HIS. The dual model approach to information modelling has been gaining traction as a strategy tackle this problem. In fact, separating the (more stable) core - the reference model - from the (ever changing) clinical model, grants the healthcare professionals more freedom to adjust the EHR to their specific and evolving needs, while preserving the structure of the HIS and unloading the IT team.

According to Garde et al., Fig. 1 reveals that "archetypes are syntactically interoperable this is ensured by the reference model and semantically interpretable this is ensured by archetypes themselves. However, archetypes alone do not ensure semantically interoperable systems and archetype development must be coordinated through "Domain Knowledge Governance to avoid incompatible archetypes for the same concept" [10].

The Pahl's research work compares two dual-model standards, namely ISO (International Organization for Standardization) 13606 and openEHR. Both have their similarities as they use a two-level domain modelling, based on the AOM to characterize their clinical content. However, the archetypes developed by each of them are very different and are not compatible. Moreover, their RMs are very different as well as their conceptual structures [11]. Figure 2 represents a schematic representation of the only open specification available (openEHR), compared with ISO 13606 and the HL7 CDA specification, attending to all requirements for a specific propose. Additionally, ISO 13606 integrates with the openEHR AOM.

ISO 13606 and openEHR can be distinguished between their purposes. While ISO 13606 is more focused in data communication, openEHR prioritizes data persistence $[12,13]$. Henceforth, due to the case study's main objective of implementing an interoperable system in a pandemic situation, the persistence and consultation of data proves to be important in monitoring the evolution of the pandemic and the discovery of new knowledge, revealed
Fig. 1 Levels of Interoperability Achieved Using an openEHR Approach. Based on [10]

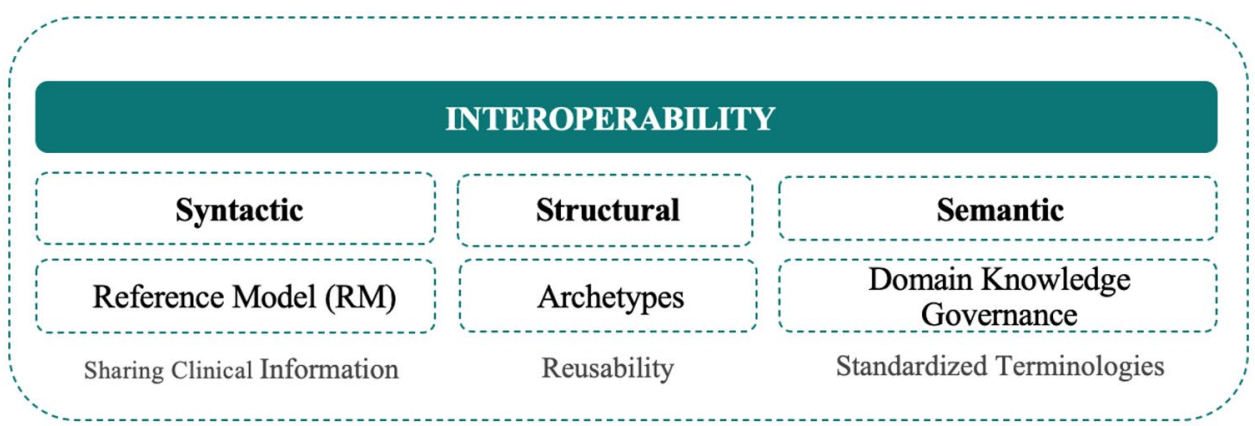


Fig. 2 Relationship between openEHR, CEN 13606, and HL7 CDA. Adapted by [11]

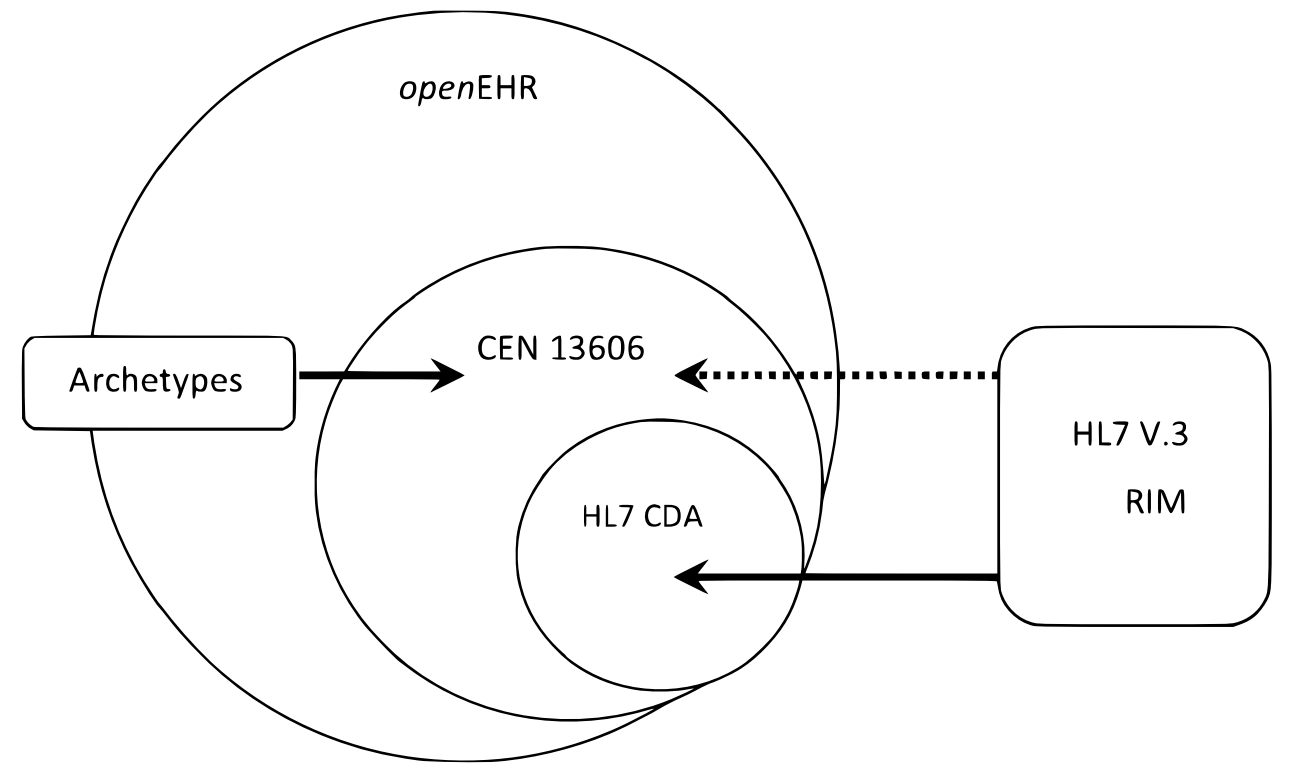

from the data produced. Thus, openEHR was the adopted standard to use and will be briefly described in the following section.

\subsection{OpenEHR}

OpenEHR is based on a dual approach separating information from domain knowledge, allowing clinical expertise define how to capture reality without the need to redefine information model that is stable. Information is represented through RM, while knowledge is streamlined through AOM $[10,14]$. The RM ensures that contextual meta-data in health records is taken care of and does not need to be redefined by each archetype. Also, the AOM presents the clinical concepts in a combined, structured, and restricted way to the entities contained in the RM $[15,16]$. Fig. 3 shows an overview of the openEHR architecture implementation as well as its different domains and each performer type. In a knowledge domain level, archetypes and templates are developed on a specific platform by clinical experts. This modelling process acts as a well-defined semantic gateway to terminologies, classifications and clinical guidelines. On the other hand, the IT specialist is responsible for the software coding with the RM and its integration with the AOM. The distributed system approach allows an easy development of one or more healthcare systems and their GUIs, with different kind of users [11, 12].

In terms of data protection, an openEHR approach ensures data resilience, maintaining them in the system in a historic and revision approach. Each change in the patient's EHR is saved through the audit system, which offers a high reliability to the structure. Finally, the patients' clinical data is separated from their administrative data, ensuring the patient's confidentiality [16, 17].

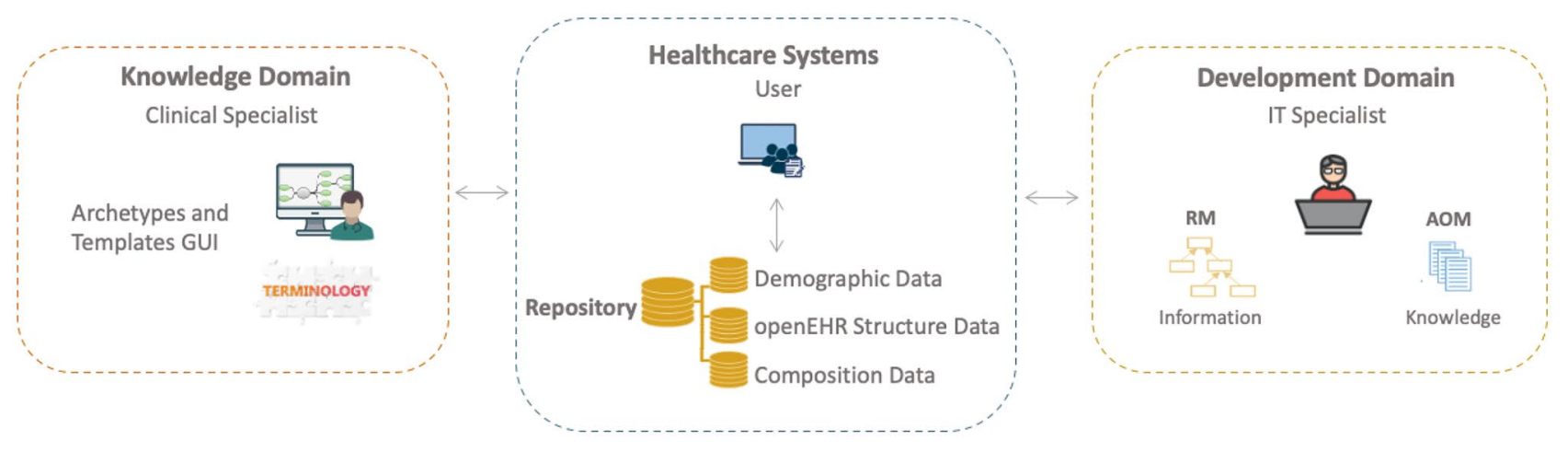

Fig. 3 openEHR Dual Architecture Scheme 


\subsubsection{Main Goals, Features, and Benefits}

Consistently modelling health information according to the openEHR specification allows the establishment of semantic interoperability between HISs and within them, avoiding data blocking by data providers. Moreover, it is an open standard, allowing everyone to reuse clinical model defined and improve it. Its documentation is complete and is constantly updated by the community.

On a technical approach, the separation of the openEHR architecture also allows for a better division of tasks during its implementation. Therefore, it is straightforward to identify the kind of professionals that will be responsible for each tier of the information model: the technical RM and the clinical AOM. The clinical experts' collaboration is extremely important, as it allows for a continuous upgrade of the AOM's structures and a constant evolution in the embedded knowledge. This possibility is supported by openEHR's versioning system, without invalidating reuse of previously used structures.

Archetypes and templates are the main artifacts of openEHR. They are multilingual and complemented with international terminologies, avoiding unnecessary processing of data. Therefore, as terminologies are global standards, the retrieval information process is semantically effective and independent of any system [18]. The RM is stable and sustainable by IT experts. It is completely independent of software applications and technologies, enabling lifelong and application independent EHRs. This approach supports many features required by complex clinical record maintenance, such as record versioning, an audit system , strong and complex structures of encounters and events, a specific AQL, and reliable data safety tools [19].

\subsubsection{Implementations}

In 2013, the article "Survey of openEHR storage implementations" reported that there were already around 16 projects based on an openEHR implementation. Their results had revealed that 11 in 16 projects have adopted a RDBMS for persistence and querying. The study also discloses that 7 of the projects had a non-commercial licensing. Furthermore, most APIs were developed using SOAP and the REST architecture. At the time of publishing, some of those systems were under development, while others were still in experimental phases, and some of them in production [20]. Hence, after 7 years, several projects were developed based on openEHR approaches in the most diverse areas of healthcare. However, there are still few research projects published on this subject applied to the COVID-19 pandemic.

In Sweden, a system called VERA - Virtual Emergency Room Administrator - was developed and consists of a prototype for an electronic emergency record using the openEHR standard and the EHRScape cloud service. Although this work did not appear in the context of the pandemic, it is adaptable in an emergency context with COVID-19 openEHR models [21]. Also, in India, the authors of a blockchain study in Healthcare context "reviewed the major uses of blockchain in clinical data security and management". They defended the use of blockchain in healthcare to achieve secure storage and integrity protection, privacy and ownership of data, data sharing, traceability, and accountability of data. Finally, in Germany, a study initiated an open-access platform for data collection of patients with COVID-19, helping conducting clinical trials for new diagnostic and therapeutic options [22]. Concerning the modelling process of openEHR structures, the collaboration of international institutions had recommended a methodology on how to develop an openEHR template from the collected data items according to COVID19 guidelines, and how to review and release the created template. As a result, the template has been uploaded into the CKM platform with the name "COVID-19 Pneumonia Diagnosis and Treatment [7th edition]" [23].

\subsection{Motivation}

As previously mentioned, this unexpected pandemic had instigated the collapse of healthcare systems in several countries, warranting a restructuring of institutions at practically all levels. In Portugal, the first COVID-19 case was diagnosed at the Centro Hospitalar Universitário do Porto in early March 2020. Facing a crisis of unknown proportions, the institution had decided to develop a add-on to the implemented HIS, based on the openEHR specification, supporting the workflow associated with suspected and confirmed cases, in order to quickly respond to the local growth of the pandemic. Resourcing to a multidisciplinary team of researchers and healthcare professionals developing the future EHR of CHUP, the process started with a frontline process mining and its subsequent openEHR modelling. This case study aims to answer several research questions raised during the implementation of new approaches in HIS. These research questions include:

RQ1: What is the relevance of using global clinical standards to promote Interoperability in Healthcare Institutions?

RQ2: Is openEHR feasible for designing and developing a new clinical workflow in a pandemic situation?

RQ3: How to integrate the new workflow developed in the institution's Information Systems?

\section{Methods}

To develop well-founded templates that can support the healthcare process and store all relevant patient information, a methodology on how to develop and support these structures 
Fig. 4 Modelling method for development of openEHR structures

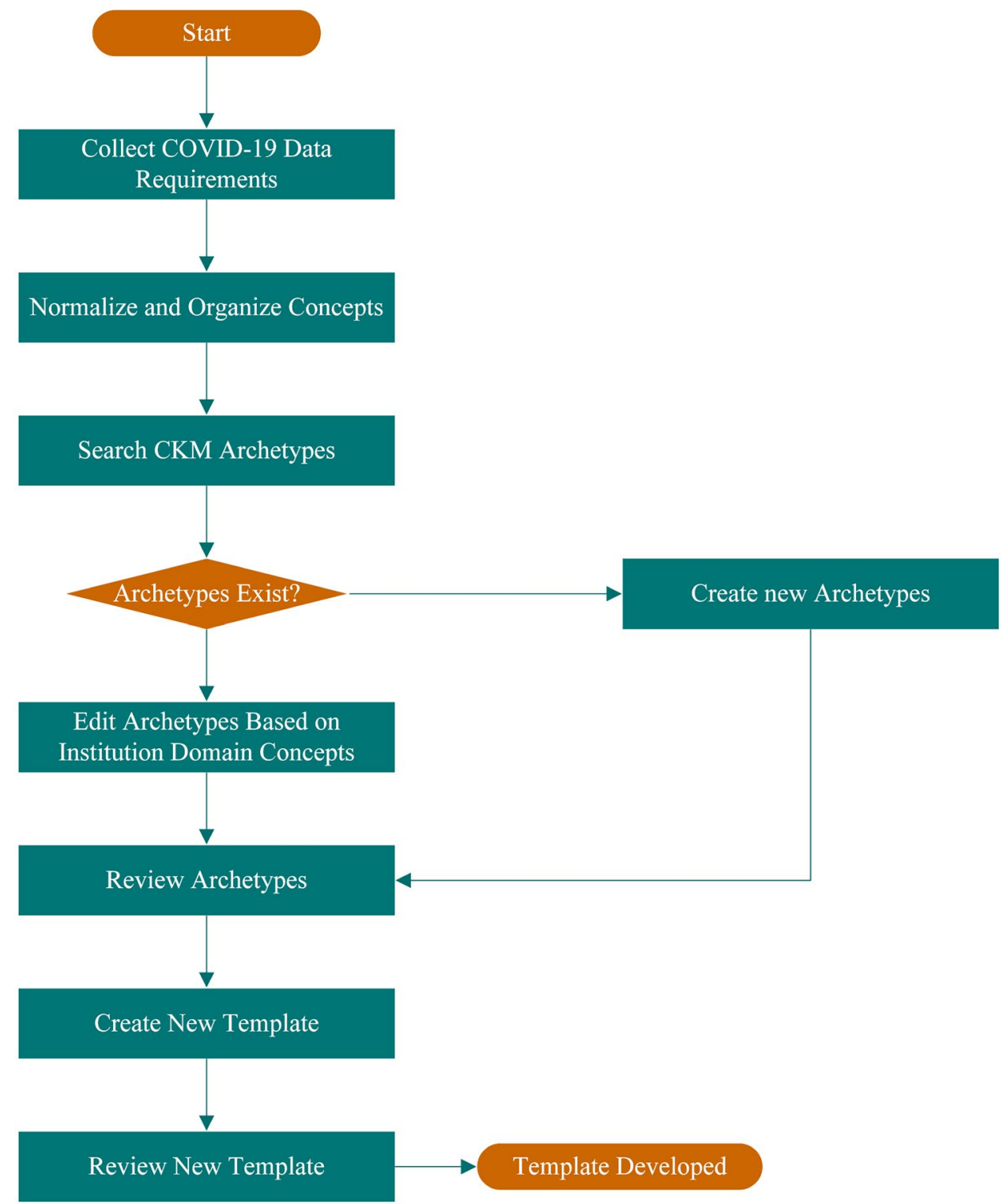

was defined and adopted (Fig. 4). As knowledge regarding COVID-19 was starting to be shared among the medical community, a requirement elicitation process allowed for the identification of the concepts to store, and how to represent them. Regarding modeling, queries on the openEHR CKM had returned useful structures, and new COVID-19-specific archetypes and templates developed by the community complemented them. From that basis, the modelling tool used was the Archetype Designer by Better in partnership with the international openEHR community [24].

In order to collect the domain concepts for modeling, it was necessary to analyze the institution's needs at the administrative and clinical levels. Throughout the process, communication between the CHUP institution and patients was constant and direct, through text messages and phone calls. The workflow of health professional's workflow during the pandemic was thus derived and shown in Fig. 5.

In the first wave, access to hospital due to suspect for COVID-19 were mainly controlled by SNS24, through telephone referral. Patients were assessed in COVID-19 screening area and sent to home after collecting sample to SARS-CoV2 when their clinical condition permitted. Results were sent by SMS when negative to SARS-CoV2 or shared by phone if the first method wasn't successful. Patients with a positive result, was assessed by phone regarding disease severity and sent to home-based care or sent to the hospital for additional study and possible admission to ward. 


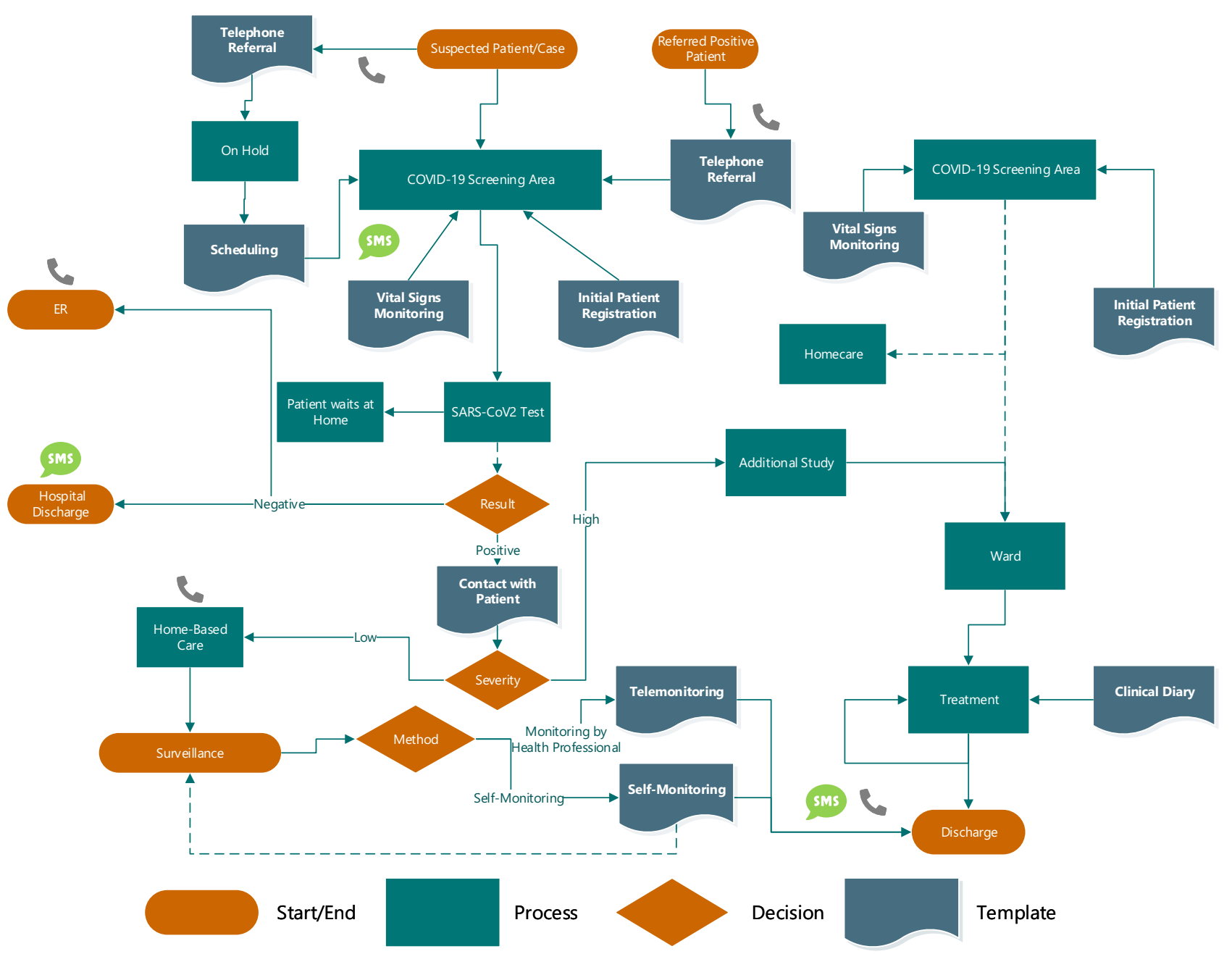

Fig. 5 Patient Treatment Flow COVID-19 in CHUP

Surveillance of patients with home-based care was carried out through self-monitoring complemented by active monitoring by health professional.

\section{Results}

The adoption of a new system based on the openEHR specification has allowed health professionals to optimize and automate their medical workflows, especially during the COVID-19 pandemic. Multiple archetypes hosted in CKM were used, witch demonstrate the importance of sharing clinical models.

Furthermore, a platform for patient monitoring and referral was developed, including web applications and a microservice-based infrastructure. Since the implementation of the proposed system and its COVID-19 workflow, thousands of compositions were submitted and processed. To present the project's KPIs, several metrics of the platform's usage were gathered and analyzed using data visualization software, namely Tableau Desktop.

The results presented are subdivided in terms of modelling process and the extraction of clinical knowledge. In order to support the proposed patient treating flow monitor, monitoring them during their recovery, multiple templates were developed. On Fig. 6 a representation of which archetypes were used in each created template is presented.

The developed templates were used to support a platform for monitoring patients during the COVID-19 pandemic. Figure 7 shows the total number of COVID-19 openEHR records submitted during the first wave, from March to June 2020.

Then, Fig. 8 presents the number of submitted templates from February to May, per template. The majority of the submitted templates are related to the developed self-monitoring application, where confirmed positive patients were able to submit their symptoms. As patients were reminded two times per day by text message, a high number of submitted templates was expected. 


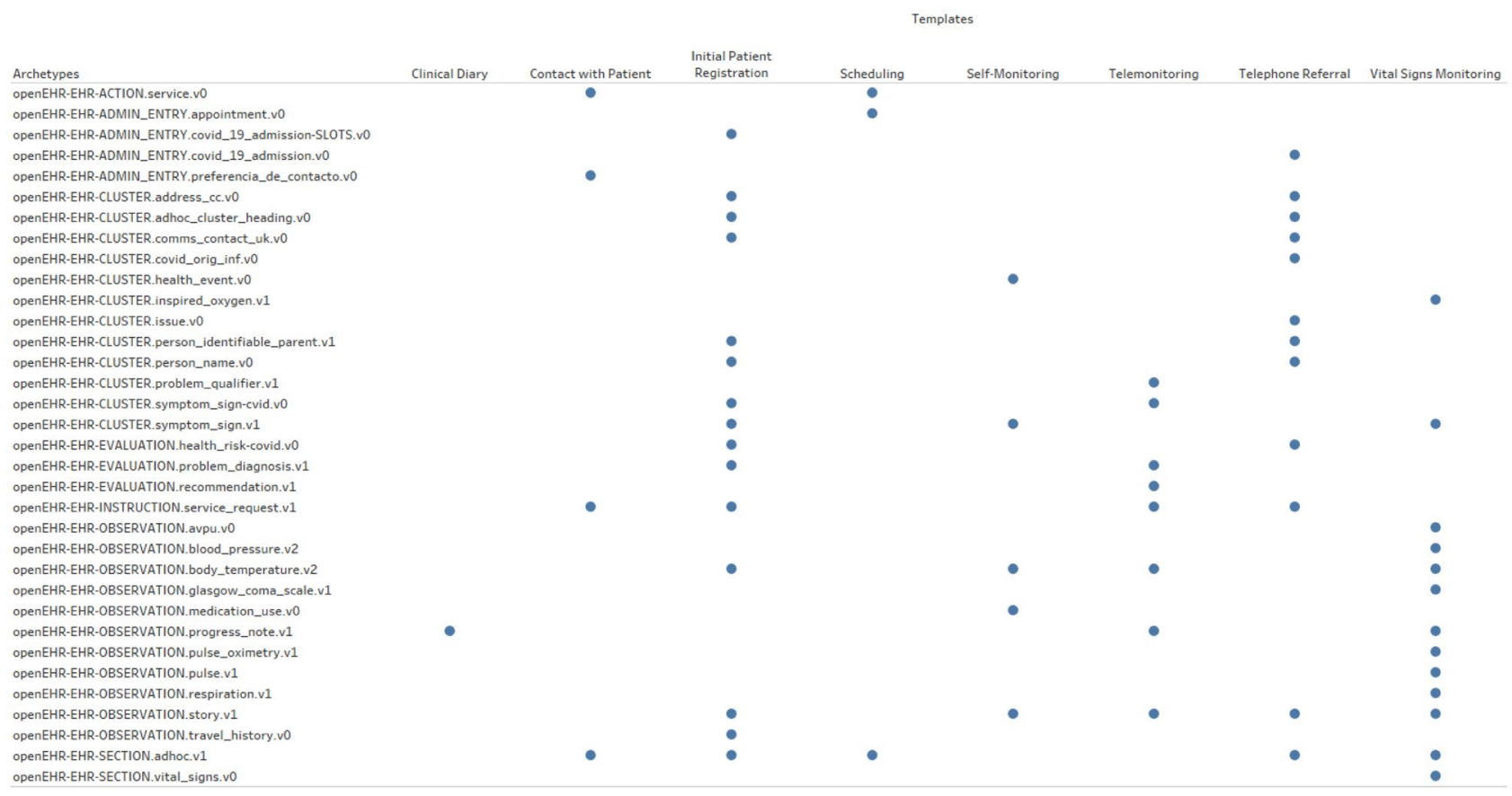

Fig. 6 Representation of the archetypes used in the created templates

By using the date range between the "Initial Patient Registration" and the "Telephone Referral" templates, it is possible to calculate how much time a patient had to wait for their COVID-19 test results. Figure 9 shows how the average waiting time for the COVID-19 test varies since it was performed.

\section{Discussion}

With the dual architecture implemented, it was possible to quickly obtain performance to reuse international data models devolepd in the OpenEHR comunity and adjust to the local needs, with a minimal effort.

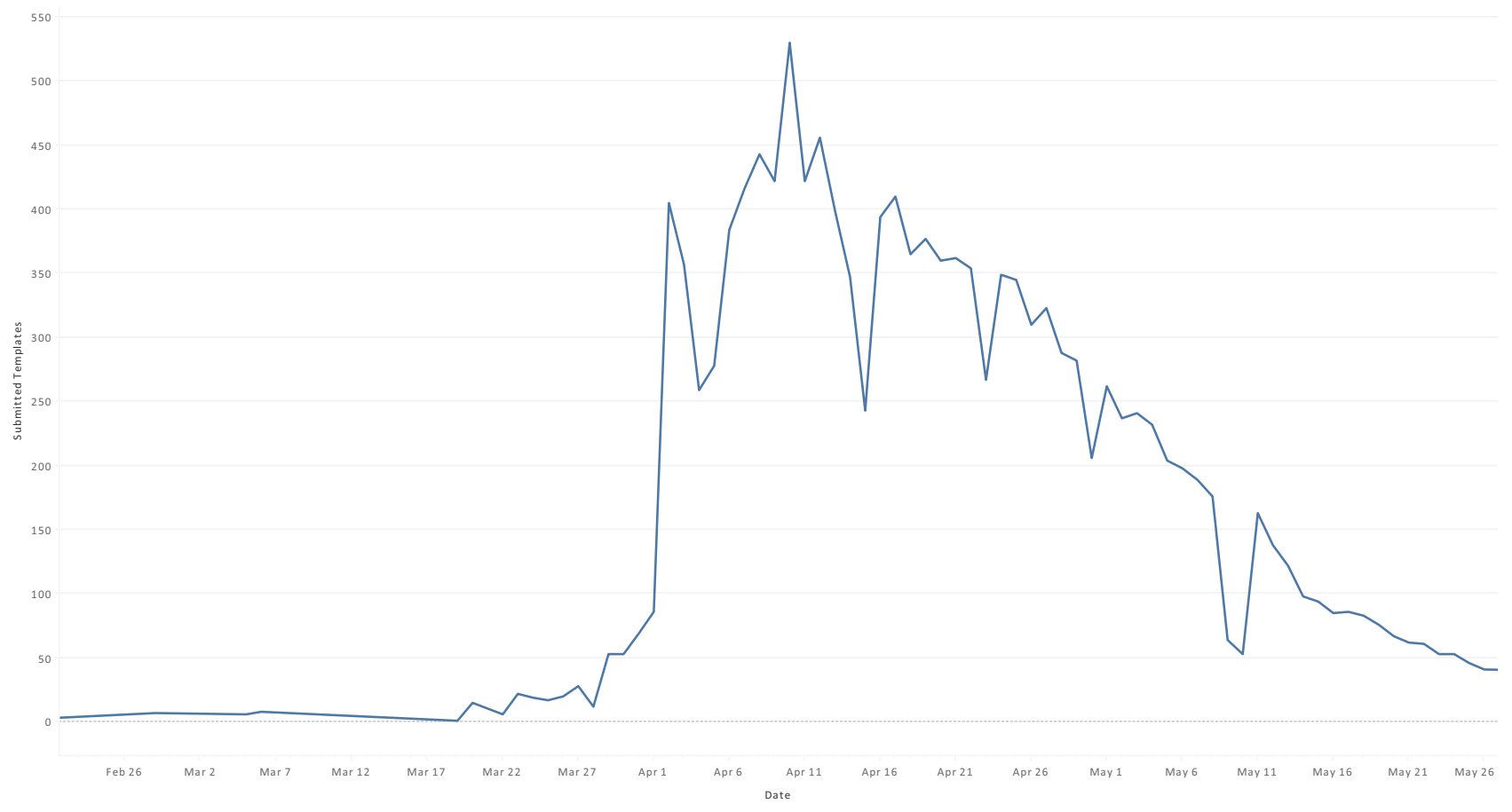

Fig. 7 Records submitted based on the openEHR structures developed 


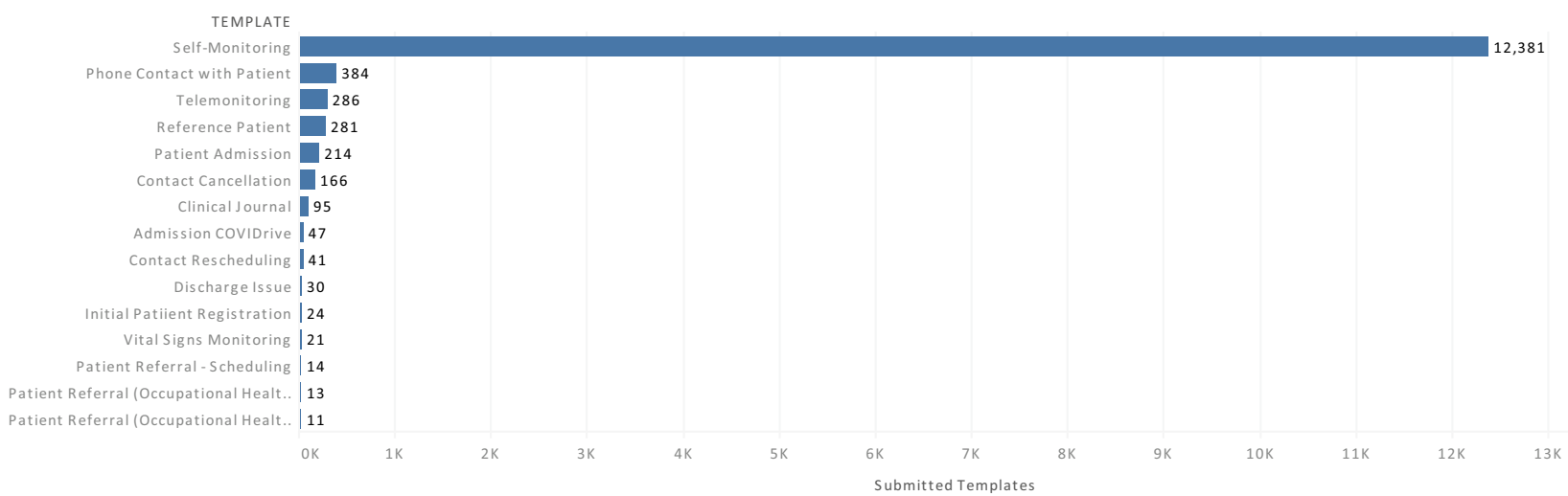

Fig. 8 Submitted Templates per template

This approach allowed the reuse of archetypes in different templates throughout the COVID-19 patient care workflow.

In addition, these templates have been adopted in different contexts, confirming a careful and extensive clinical modelling.

The most rewarding feature of using openEHR is its high flexibility in the face of constantly changing knowledge scenarios.
When changing clinical knowledge, it was only necessary to change the template, maintaining the uniformity of the software involved in the openEHR system.

Modelling process must be accompanied by the necessary clinical guidelines to maintain data integrity in a specific clinical context, and must always be developed as a continuous process so that its update is quick and effective.

With the results of this study, it is possible to answer the research questions proposed initially.

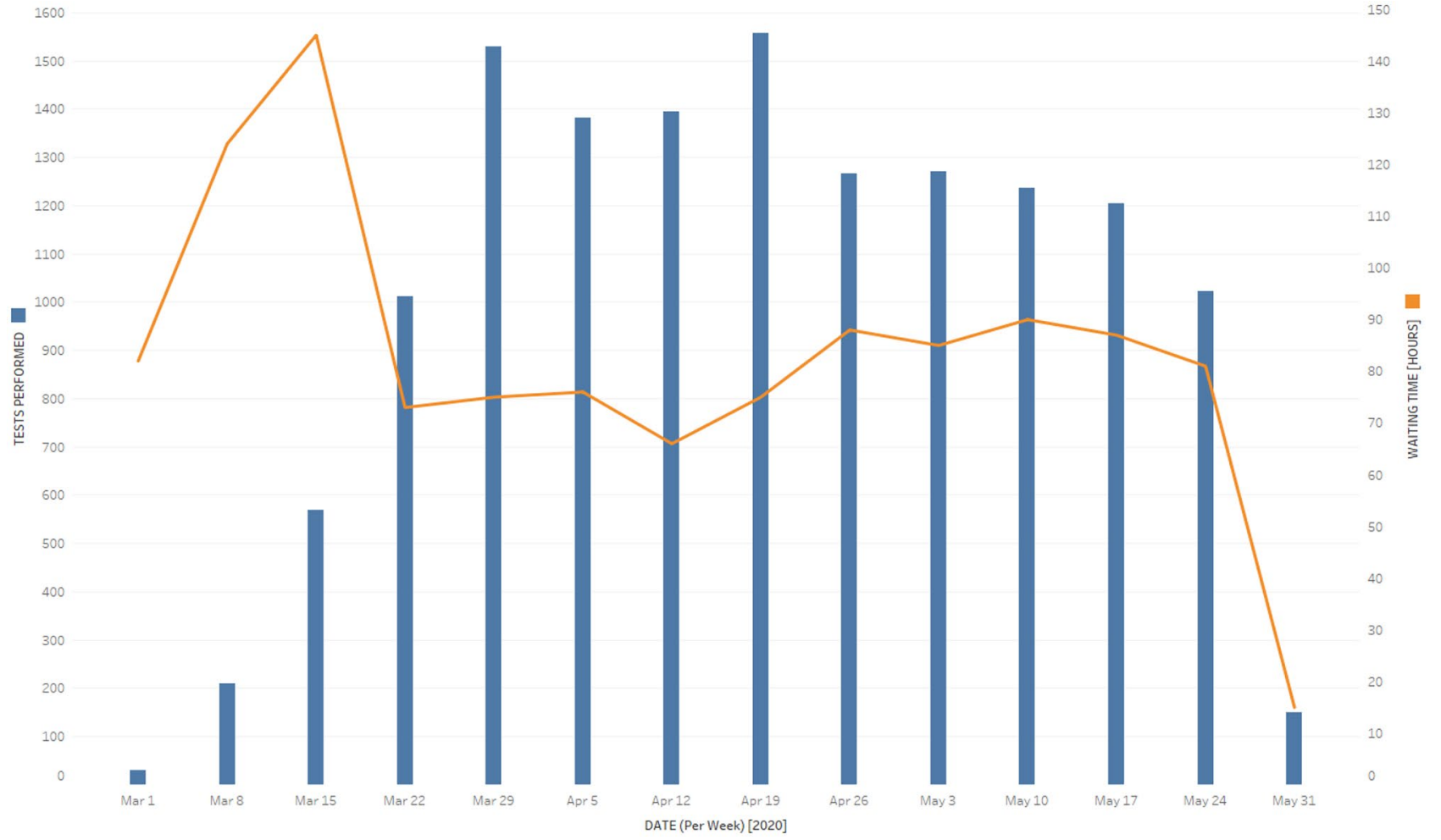

Fig. 9 Average COVID-19 Test Waiting Time 
What is the relevance of using global clinical standards to promote Interoperability in Healthcare Institutions?

The use of global standards ensures the symbiosis of reliable and ubiquitous software with other devices and components. These standards establish interoperability in different contexts, allowing communication with external applications. In a clinical domain, the use of openEHR standard allows the reuse of the clinical data model defined at an international level, with the commitment of local needs. Figure 6 reveals the potential of reusing archetypes to develop structures (templates) for specific use cases. Thus, the implementation of an international standard such as openEHR allows institutions to achieve their different levels of interoperability (semantic, syntactic and structural), ensuring the ability to use technology to share, as well as exchange data from hospitals, laboratories, pharmacies, etc. Consequently, with a high level of interoperability in healthcare institutions improves the patients' experience, shortens waiting times, improves and simplifies clinical records, decreases costs associated with previous methods as well as possible errors, preserves patient privacy, among others.

Is openEHR feasible for designing and developing a new clinical workflow in a pandemic situation?

In a pandemic situation, the clinical guidelines and clinical knowledge are constantly improving and changing, translating into a need to implement a type of architecture that allows to distribute the development process by clinical and IT experts, complementing each other. The adoption of a dual architecture approach, which separates the reference model with the archetype model, translates into a continuous improvement, with dynamic and constantly changing modelling structures. For this to be possible, it is necessary to first outline a modelling methodology that allows clinical experts to model in a consistent and reusable way. On the other hand, it is necessary to develop the workflow for each case study to model. In a pandemic case study, it is necessary to understand the first steps for the identification and tracking of the patient, modelling them. After the patient tracking, it is essential to understand the possible paths that the patient can take from the admission time. If the patient has not been discharged from hospital, the different types of hospitalization - hospital or home care - must be properly modeled and followed up until discharge.

How to integrate the new workflow developed in the institution's Information Systems?

Firstly, a dynamic GUI framework should be developed to interpret the templates modelled through the proposed modelling methodology. Subsequently, the developed templates must be transposed and visualized through the developed framework, so that they can be used later in all target IT's, both for data entry and visualization. To make this possible, mechanisms must be developed to insert and retrieve data from the different legacy systems already used in the institution, mapping them with the modelled openEHR structures.

\section{Conclusions and future work}

This case study is part of a pilot project for the implementation of openEHR at the CHUP institution. After analyzing the institution's needs, it was decided to adopt the openEHR clinical methodology, with the support of a vast international community and constantly evolving around the world. The research archetype proposed and developed was put in place during the beginning of the COVID-19 pandemic in Portugal, helping the institution and its health professionals to organize themselves in times of crisis and high pressure. The methodology also allowed the institution to be able to outline its clinical guidelines in a structured way, as well as deal with its constant changes. Based on the different results until we reached the final COVID-19 workflow and the constant feedback from users, the developed system proved to be quick to change, adapt and employ, resulting in a high gain for the institution. The system translates into dynamic flexibility associated with the current pandemic, being able to easily interoperate with other HISs. Through standardized and structured data records, it was possible to quickly obtain the most diverse indicators both in terms of their workflow and also of the development of the pandemic in the healthcare institution.

As a future work, it is necessary to identify and develop the Demographic Information Model so that it is possible to identify different attributes and relationships of demographic entities that exist independently of particular clinical involvement or participation in particular events. After that, it is inevitable to develop the EHR Information Model so that the implementation of the RM is complete.

Abbreviations API: Application Programming Interface; ADL: Archetype Definition Language; AOM: Archetype Object Mode; AQL: Archetype Querying Language; CHUP: Centro Hospitalar Universitario do Porto; CDS: Clinical Decision Support ; CKM: Clinical Knowledge Manager; EHR: Electronic Health Record; CEN: European Committee for Standardization; GUI: Graphical User Interface ; HIS: Healthcare information systems; IS: Information System ; IT: Information Technology; IHR: International Health Regulation ; ISO: International Standardization Organization; KPI: Key Indicators of Performance; MI: Medical Informatics; RM: Reference Model ; RDBMS: Relational Database Management System; REST: Representational State Transfer; SARSCoV-2: Severe Acute Respiratory Syndrome Coronavirus 2 ; SOAP: Simple Object Access Protocol ; WHO: World Health Organization

Funding Information This work has been supported by FCT - Fundacao para a Ciuencia e Tecnologia within the R\&D Units Project Scope: UIDB/00319/2020. 


\section{Declarations}

Conflicts of interest The authors declare that they have no conflict of interest

Ethical standard This article does not contain any studies with human participants or animals performed by any of the authors.

\section{References}

1. Cardoso L, Marins F, Portela F, Santos M, Abelha A, Machado J. The Next Generation of Interoperability Agents in Healthcare. Inte J Environ Res Publ Health. 2014;11(5):5349-71. https://doi. org/10.3390/ijerph110505349.

2. Duarte J, Salazar M, Quintas C, Santos M, Neves J, Abelha A, Machado J. Data Quality Evaluation of Electronic Health Records in the Hospital Admission Process. In 2010 IEEE/ACIS 9th International Conference on Computer and Information Science, pages 201-206. IEEE, 2010. https://doi.org/10.1109/ICIS.2010.97

3. Pereira R, Salazar M, Abelha A, Machado J. Swot analysis of a portuguese electronic health record. In C. Douligeris, N. Polemi, A. Karantjias, and W. Lamersdorf, editors, Collaborative, Trusted and Privacy-Aware e/m-Services, pages 169-177, Berlin, Heidelberg, 2013. Springer Berlin Heidelberg.

4. Salazar M, Duarte J, Pereira R, Portela F, Santos MF, Abelha A, Machado J. Step towards paper free hospital through electronic health record. In: Rocha A, Correia AM, Wilson T, Stroetmann KA, editors. Adv Info Syst Tech. Berlin Heidelberg, Berlin, Heidelberg: Springer; 2013. p. 685-94.

5. Wu D, Wu T, Liu Q, Yang Z. The SARS-CoV-2 outbreak: What we know. Int J Infec Dis. 2020;94:44-8.

6. Oliveira D, Coimbra A, Miranda F, Abreu N, Leuschner P, Machado J, Abelha A. New Approach to an openEHR Introduction in a Portuguese Healthcare Facility. pages 205-211. https:// doi.org/10.1007/978-3-319-77700-921

7. Peixoto H, Santos M, Abelha A, Machado J. Intelligence in Interoperability with AIDA. pages 264-273. 2012. http://link.springer. com/10.1007/978-3-642-34624-831

8. CEN. Standards make your life safer and simpler. https://www. cen.eu/you/Pages/default.aspx

9. Bauer M, Bilbao S, Daniele L, Esnaola-Gonzalez I. Towards Semantic Interoperability Standards. (October):0-26, 2019. https://doi.org/10.13140/RG.2.2.26825.29282
10. Garde S, Knaup P, Hovenga EJ, Heard S. Towards semantic interoperability for electronic health records: Domain knowledge governance for openEHR archetypes. Meth Info Med. 2007;46(3):332-43. https://doi.org/10.1160/ME5001.

11. Pahl C, Zare M, Nilashi M, de Faria Borges MA, Weingaertner D, Detschew V, Supriyanto E, Ibrahim O. Role of OpenEHR as an open source solution for the regional modelling of patient data in obstetrics. J Biomed Info. 2015:174-187. http://dx.doi.org/10. 1016/j.jbi.2015.04.004

12. Martínez-Costa C, Menárguez-Tortosa M, Fernández-Breis JT. Towards ISO 13606 and openEHR archetype-based semantic interoperability. Stud Health Tech Infor. 2009;150:260-4.

13. McNicoll I. openEHR and ISO/CEN 13606 - what is the relationship? https://discourse.openehr.org/t/openehr-and-iso-cen-13606what-is-the-relationship/224

14. Schuler T, Garde S, Heard S, Beale T. Towards automatically generating graphical user interfaces from openEHR archetypes. Studies in health technology and informatics, pages 221-6. http:// www.ncbi.nlm.nih.gov/pubmed/17108529

15. Beale T, Heard SOI. Archetype Definitions and Principles. Ocean Informatics, page 15, 2007.

16. openEHR Foundation. openEHR - Design Principles, 2017.

17. EtherCIS. Introducing openEHR. http://docs.ethercis.org/ openEHRintro.html

18. openEHR Foundation. openEHR Support Terminology. 2020. https://specifications.openehr.org/releases/TERM/latest/ SupportTerminology.html

19. Leslie H. A. Informatics. openEHR - the World's Record. ResaerchGate, (June):10, 2015.

20. Frade S, Freire SM, Sundvall E, Patriarca-Almeida JH, Cruz-Correia RJ. Survey of openEHR storage implementations. Proceedings of the 26th IEEE International Symposium on Computer-Based Medical Systems, pages 303-307, 2013.

21. Homssi R, Lindgren F, Hasselquist HD. VERA - Virtual Emergency Room Administrator. 2020.

22. Salg GA, Ganten MK, Baumhauer M, Heussel CP, Kleesiek J. A globally available COVID-19 - Template for clinical imaging studies. medRxiv, page 2020.04.02.20048793. 2020. https://doi. org/10.1101/2020.04.02.20048793

23. Li M, Leslie H, Qi B, Nan S, Feng H, Cai H, Lu X, Duan H. Development of an openEHR Template for COVID-19 Based on Clinical Guidelines. J Med Int Res. 2020;22(6):e20239.

24. Better. Archetype Designer. https://tools.openehr.org/designer/\#/ 